\title{
Modeling of IPMC (Ionic Polymer-Metal Composite) Sensor to Effectively Detect the Bending Angles of a Body
}

\author{
Kiwon Park ${ }^{+}$
}

\begin{abstract}
Ionic polymer-metal composite(IPMC) consists of an ion conductive membrane plated by metallic electrodes on both surfaces. When it bends, a voltage is generated between two electrodes. Since IPMC is flexible and thin, it can be easily mounted on the various surfaces of a body. The present study investigates a sensor system using IPMC to effectively detect the bending angles applied on IPMC sensor. The paper evaluates several $\mathrm{R}$ and $\mathrm{C}$ circuit models that describe the physical composition of IPMC and selects the best model for the detection of angles. The circuit models implemented with a charge model describe the relationship between input bending angles and output voltages. The identification of $\mathrm{R}$ and $\mathrm{C}$ values was performed by minimizing the error between the real output voltages and the simulated output voltages from the circuit models of IPMC sensor. Then the output signal of a sensor was fed into the inverse model of the identified model to reproduce the bending angles. In order to support the validation of the model, the output voltages from an arbitrary bending motion were also applied to the selected inverse model, which successfully reproduced the arbitrary bending motion.
\end{abstract}

Keywords : IPMC Sensor, Charge Model, Circuit Model, Parameter Estimation, Model Identification

\section{INTRODUCTION}

IPMC consists of an ion-exchange-polymer membrane and metal electrodes. The polymer membrane is only permeable to the cations while anions are fixed at the backbone polymer. The polymer membrane is chemically plated or electroplated with metal for the electrodes. When IPMC is deformed mechanically, the solvent molecules and cations in the compressed side of membrane move toward the stretched side of membrane as depicted in Fig. 1. The imbalanced amount of cations contacting with each electrode generates output voltages across the membrane.

IPMC has great potential as a biological sensor. First, it is flexible and light; therefore it is compliant with the movement of soft object such as the movement of a muscle[1]. Second, its simple structure can be utilized in fabricating as a small size sensor[1]. Third, its sustaining performance in wet environment gives it potential to be used as an embedded biomedical sensor[2]. The characteristics of IPMC sensor have investigated by fewer researchers than those of IPMC actuator and most researches on IPMC sensor have focused on developing a model of the sensor which simulated output voltages or output currents[1-5]. A

Biomedical Engineering, South Dakota School of Mines and Tech. South Dakota, USA

+Corresponding author: kiwon.park@mines.sdsmt.edu

(Received : June. 27, 2011, Accepted : Oct. 1, 2011) main assumption of the models was that the amount of charge density variation inside membrane is proportional to the induced strain within the polymer membrane[4, 5]. Although the proposed models successfully simulated output signals, their performances were limited to the detection of small deformations of the sensors. In addition, the complex mathematical forms of the models made it difficult to understand the dynamics of the sensor. Using a circuit model is a systematic way to understand the dynamics of the sensor and to identify the relationships between the physical and model parameters of the sensor.

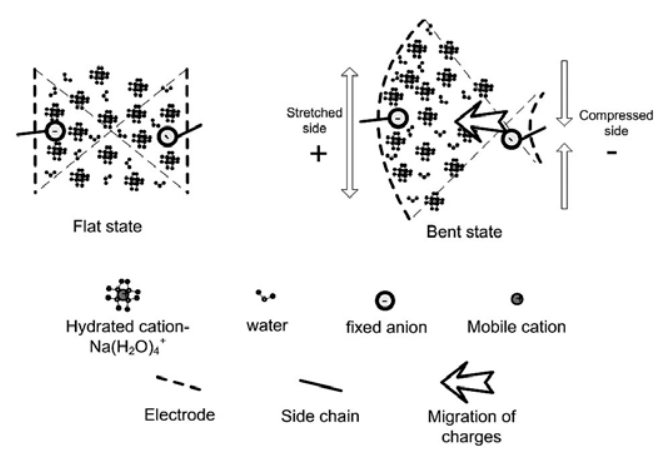

Fig. 1. Operating mechanism of an IPMC sensor.

The present study evaluates the several $\mathrm{RC}$ circuit models that describe the physical composition of IPMC and finds the best circuit 
model of IPMC as a large bending sensor. The parameters of each model were identified such that the root mean square(RMS) error between the real and simulated output voltages was minimized. After the parameters were identified, the effectiveness of models was tested by comparing the errors and the shapes of simulated output voltages from each model. The model evaluation was also conducted by comparing the input angles with the reproduced angles from the inverse transfer function of the circuit models. After the best model was selected, the output voltages from an arbitrary bending motion of the sensor were fed into the inverse transfer function of the selected model. The inverse model successfully reproduced the arbitrary bending motion.

\section{EXPERIMENTAL DESIGN}

\subsection{Fabrication of IPMC}

IPMC was fabricated using a Nafion ${ }^{\circledR} 117$ membrane with a thickness of $178 \mu \mathrm{m}$ and platinum particles. The particles were electrolessly plated on both sides of the membrane by a method proposed by Dr. Oguro[9]. The fabrication procedure has following steps: (a) cleaning of the Nafion ${ }^{\circledR}$ film using HCL solution and deionized water(DI water), (b) adsorption of platinum cations to be bonded with anion groups on the Nafion ( ${ }^{\circledR}$ film. (c) primary plating using sodium borohydrate $\left(\mathrm{NaBH}_{4}\right)$ as a reduction chemical. (d) additional plating using solutions of hydroxylamine hydrochloride $\left(\mathrm{NH}_{2} \mathrm{OH}-\mathrm{HCl}\right)$ and hydrazine $\left(\mathrm{NH}_{2} \mathrm{NH}_{2}\right)$ as additional reduction chemicals. After the fabricated IPMC was cleaned with DI water, the IPMC was cut into a size of $20 \mathrm{~mm} \times 3 \mathrm{~mm}$ for the experiment. Fig. 2 shows the fabricated IPMC sensor.

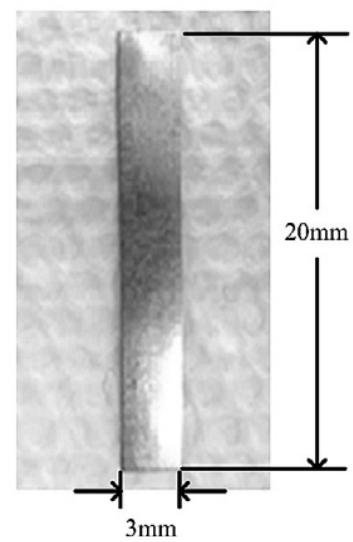

Fig. 2. Fabricated IPMC sensor.

\subsection{Experimental setup}

Fig. 3 shows a schematic diagram of the output signal measurement system. One end of IPMC was fixed by a plastic clamp. The output voltage across the IPMC sensor was measured through the copper electrodes inside the clamp. A guider, which was fixed on the rotational disc of a stepper motor, slightly held the opposite end of IPMC. Since the guider located $15 \mathrm{~mm}$ away from the center of the disc, the rotation of disc applied bending motion to the IPMC. The rotation of disc was controlled by the stepper motor by a step angle of $1.8^{\circ}$. The bending angle of IPMC was defined by $\theta_{B}$ in Fig. 3 to describe the tip displacement of IPMC strip. Note that the bending angle is proportional to the rotation angle of the stepper motor shaft. The data of bending angles were also measured simultaneously by a potentiometer which was placed at the opposite end of the motor shaft.

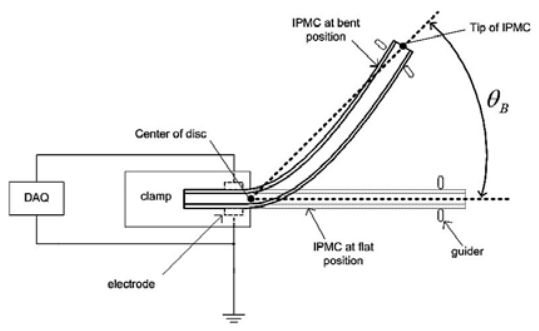

Fig. 3. Experimental setup for the output signal measurement; $\theta_{B}$ is the bending angle.

The stepper motor was controlled by a microprocessor(PIC 16F876, Microchip) which interfaced with PC via a serial port. The output voltage of the sensor was amplified by 100 times and acquired with the bending angle data from the potentiometer via a data acquisition board(NI USB-6211, National Instruments) at a sampling rate of 100 samples per second. Matlab ${ }^{\circledR}$ was used to send a trigger signal to the stepper motor to start cyclic bending motion of the IPMC sensor. Matlab ${ }^{\circledR}$ was also used to control the data acquisition board and to post-process the collected data. The experiments were conducted in air. Fig. 4(a) illustrates the applied input bending motion. In a cycle, 11 different bending angles from $3.6^{\circ}$ to $90^{\circ}$ were applied by the guider. The bending speed of motor was fixed at $150^{\circ}$ per second. Once the IPMC was bent by an angle, the motor was stopped for $0.5 \mathrm{sec}$, and then rotated backward to $0^{\circ}$. The motor was also stopped between each bending motion for $0.5 \mathrm{sec}$. A complete bending cycle was finished in $30 \mathrm{sec}$. Fig. 4(b) describes an example of the output signal of IPMC sensor with the applied bending cycle. 
The output signal has inherent offsets. There were several approaches to remove the offsets such as using a high pass filter with a low cutoff frequency usually less than $0.05 \mathrm{~Hz}[1,3]$. However a few preliminary tests showed that using a high pass filter may significantly change the output characteristics of IPMC sensor. For this reason only amplified output signal was measured without the filter. In order to eliminate the offsets from the output signal, the magnitude of initial offsets depicted in Fig. 4(b) was subtracted from the original output signal.

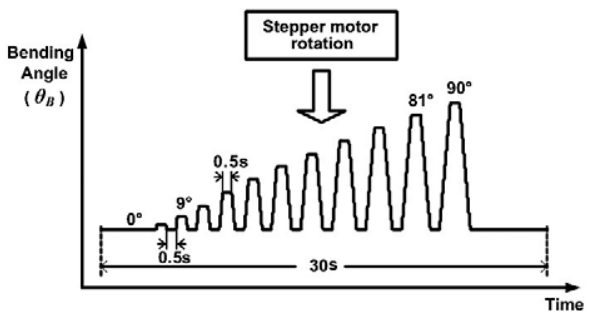

(a)

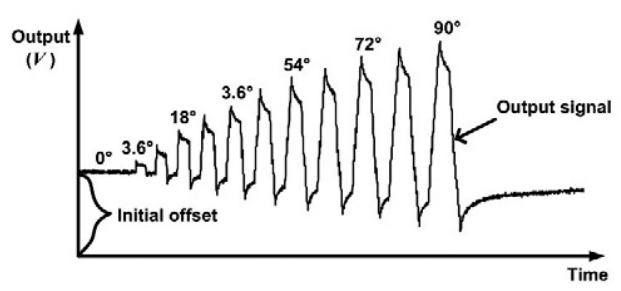

(b)

Fig. 4. Output signal with respect to the input bending angles; (a) illustration of the applied bending angles and (b) the corresponding output signal and offset.

\subsection{Modeling of an IPMC sensor}

Many researches on developing IPMC sensor models have presented a key assumption that the induced total charge inside membrane is proportional to the applied deformation of the sensor $[4,5]$. In the present study we represent the relationship between the induced total charge $(q)$ and the bending angle $(\theta)$ of IPMC sensor by using a constant gain $(K)$. The expression for a bending angle to the induced charge is given by Eq. (1).

Therefore the expression for a bending angle to the current generated inside membrane can be given by Eq. (2).

$$
\begin{gathered}
q=K \theta_{B} \\
I=K \frac{d \theta_{B}}{d t}
\end{gathered}
$$

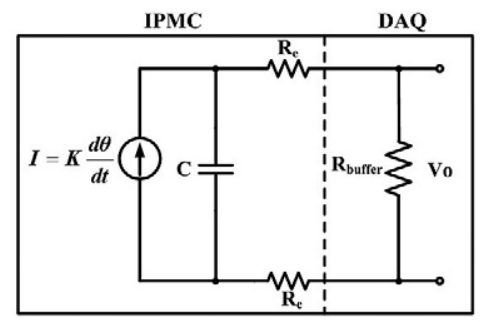

(Model 1)



(Model 2)

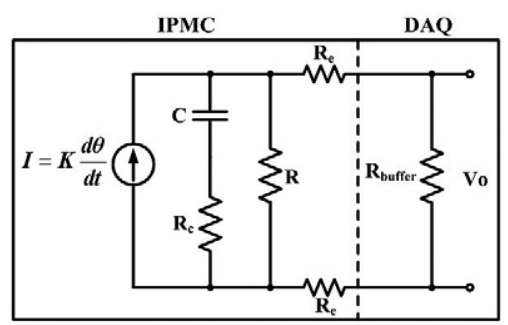

(Model 3)

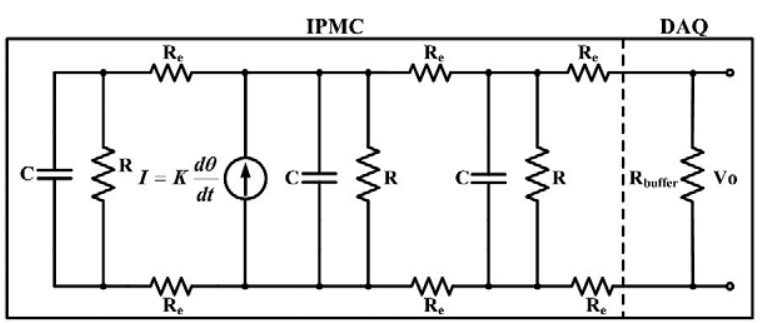

(Model 4)

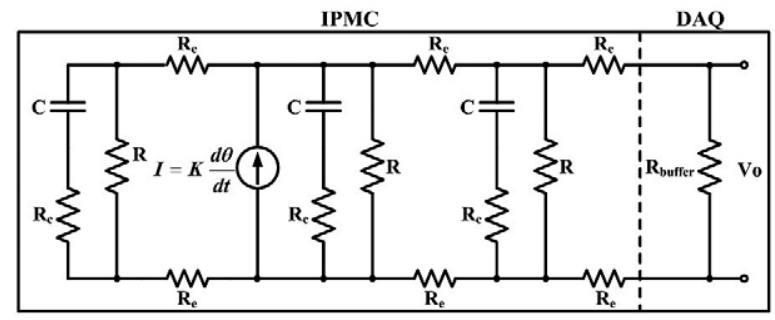

(Model 5)

Fig. 5. Candidates of IPMC sensor circuit model; (Model 1) A simple model of piezoelectric material, (Model 2) the model suggested by Shahinpoor et al.[6], (Model 3) the model suggested by Kanno et al.[7], (Model 4) the cascade connection of Model 2, and (Model 5) the cascade connection of Model 3. 
Fig. 5 shows a few circuit models of an IPMC sensor. Models 1, 2, and 3 can be categorized into the unit-circuit models. Models 4 and 5 can be categorized into the cascade-circuit models which consist of three unit-circuit models connected in series. Each circuit model is connected to the input resistance $\left(\mathrm{R}_{\text {buffer }}\right)$ of a buffer circuit for the measurement device through the electrode resistances $\left(\mathrm{R}_{\mathrm{e}}\right)$. Model 1 describes a simple model of piezoelectric material that consists of a capacitance $(\mathrm{C})$ and an electrode resistance $\left(\mathrm{R}_{\mathrm{e}}\right)$. The polymer membrane between the two electrodes acts as the capacitance. Model 2, suggested by Shahinpoor et al.[6], consists of three components, a capacitance $(C)$, a polymer resistance $(R)$, and an electrode resistance $\left(\mathrm{R}_{\mathrm{e}}\right)$. Model 3 was suggested by Kanno et al.[7]. The main difference between Model 2 and Model 3 is that Model 3 has an additional resistance $\left(\mathrm{R}_{\mathrm{c}}\right)$ connected in series to a capacitance. The unit-circuit model located at the middle of the cascade models(Models 4 and 5) represents the deformed part of IPMC sensor in a cantilever configuration. The other two unit-circuit models in the cascade models represent the undeformed part close to the clamp and moving tip, respectively, of the IPMC sensor that is at bent position. The circuit models were built and tested by the circuit modeling tool, SimPowerSystems in Matlab ${ }^{\circledR}$. The parameter identification was conducted by minimizing the RMS error between the output signal from a real IPMC sensor and the simulated output signal from the circuit model as shown in Fig. 6. During the estimation procedure, the electrode resistances were set to $5 \Omega$ in Models 1, 2, and 3 and $5 / 3 \Omega$ in the cascade models. The input resistance $\left(\mathrm{R}_{\text {buffer }}\right)$ of the buffer circuit was set to $1 \mathrm{G}$ $\Omega$ in all models.

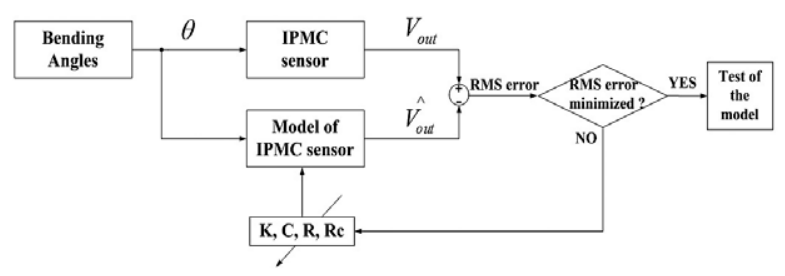

Fig. 6. Block diagram for parameter identification.

After the parameters were identified, the transfer function $(M)$ of each circuit model was calculated using the command "power_analyze" in Matlab ${ }^{\circledR}$. In order to reproduce the applied input bending angles, an inverse transfer function $\left(M^{-1}\right)$ of the sensor model was calculated and implemented to the estimation procedure. Fig. 7 shows the procedure of bending angle estimation using the inverse transfer function $\left(M^{-1}\right)$ of the IPMC sensor model.

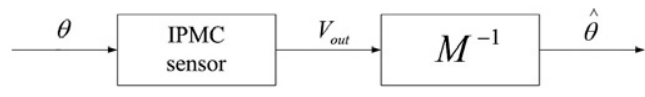

Fig. 7. Block diagram for bending angle estimation.

\section{RESULTS AND DISCUSSIONS}

\subsection{Output signal estimation}

Table 1 shows the estimated parameters obtained from the parameter identification procedure described in the previous section and the RMS errors in the output voltages between the models and the sensor. Model 3 and the corresponding cascade model, Model 5, show the smallest errors.

Table 1. Estimated parameters of the circuit models

\begin{tabular}{llllll}
\hline Parameters & Model 1 & Model 2 & Model 3 & Model 4 & Model 5 \\
\hline $\mathrm{C}$ & $1.17 \times 10^{-8} \mathrm{~F}$ & $2.10 \mathrm{mF}$ & $3.10 \mathrm{mF}$ & $2.10 \mathrm{mF}$ & $3.10 \mathrm{mF}$ \\
$\mathrm{R}_{\mathrm{c}}$ & & & $5.01 \times 10 \Omega$ & & $5.01 \times 10 \Omega$ \\
$\mathrm{R}$ & & $5.52 \mathrm{~K} \Omega$ & $4.42 \mathrm{~K} \Omega$ & $5.52 \mathrm{~K} \Omega$ & $4.42 \mathrm{~K} \Omega$ \\
$K$ & $4.59 \times 10^{-13}$ & $8.16 \times 10^{-8}$ & $1.20 \times 10^{-7}$ & $2.44 \times 10^{-7}$ & $3.60 \times 10^{-7}$ \\
RMS error & $4.27 \times 10^{-2}$ & $4.27 \times 10^{-2}$ & $1.49 \times 10^{-2}$ & $4.32 \times 10^{-2}$ & $1.48 \times 10^{-2}$ \\
\hline
\end{tabular}

Fig. 8 shows the plots of the real output voltages acquired from the sensor and the simulated output voltages from the models with the estimated parameters. Fig. 8 (a) and (b) show the output and simulated voltages obtained from Models 1 and 2, respectively. Both models show almost the same shapes of voltages and the corresponding errors are the same too(See Table 1). This is because the derived transfer functions from the two models were almost the same although Model 2 has an additional resistance(R) compared to Model 1. Note the capacitance value in Model 2 is in a proper range of capacitance value of the IPMC sensor[10].

Model 3 produces the closest simulated voltages to the real voltages. Note Model 3 simulates the peak voltages and exponential decays while Models 1 and 2 could not mimic the peaks in the output voltages.

The cascade models have the same parameter values as their corresponding unit-circuit models. Accordingly, the error values from the cascade models are almost the same as those from their unit-circuit models. However, the 
cascade models have three times greater values of $\mathrm{K}$ than the unit-circuit models. Since the identical three unit-circuit models are connected in parallel in the cascade models, the total impedance becomes one third of that of the corresponding unit-circuit models.

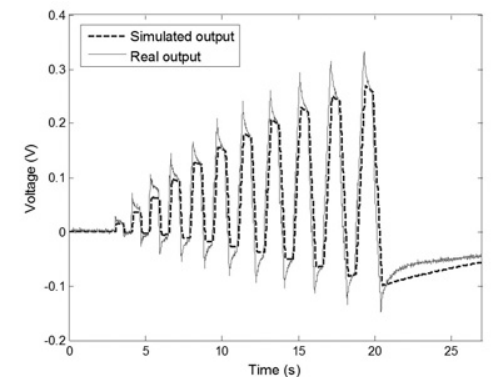

(a)

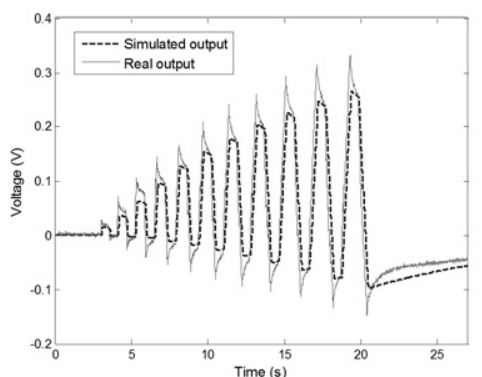

(b)

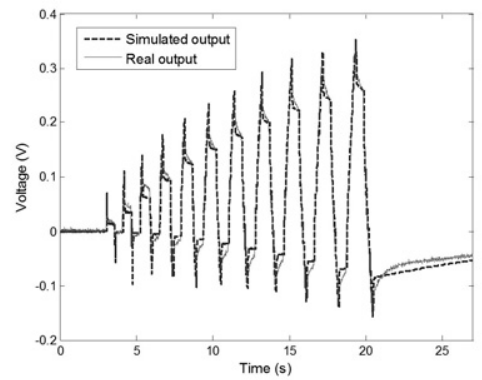

(c)

Fig. 8. Simulated outputs from the three models; (a) the simulated output from Model 1, (b) the simulated output from Model 2, and (c) the simulated output from Model 3.

\subsection{Bending angle estimation}

Fig. 9 shows the reproduced bending angles from the inverse transfer functions of Models 1,2, and 3. Table 2 shows the error values between the real input and reproduced bending angles. Since Models 1 and 2 have almost the same transfer functions, the outputs from their inverse transfer functions are very similar as shown in Fig 9(a) and (b), resulting in similar error values as shown in Table 2. In addition, the two models show high overshoots in the reproduced bending angles for the step bending angle inputs. Model 3, however, shows significantly reduced overshoots in the reproduced bending angles as shown in Fig. 9(c). Model 3 produced the smallest RMS error and Model 5 produced slightly higher one in the reproduced bending angles.

Table 2. Errors from a cycle of bending motion

\begin{tabular}{llllll}
\hline & Model 1 & Model 2 & Model 3 & Model 4 & Model 5 \\
\hline RMS error & 10.99 & 11.15 & 2.38 & 11.33 & 2.41 \\
\hline
\end{tabular}

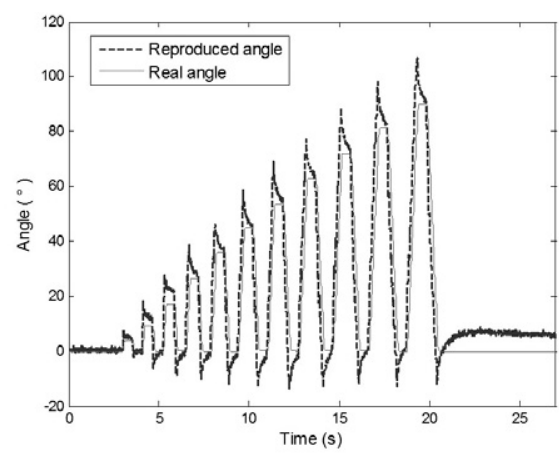

(a)

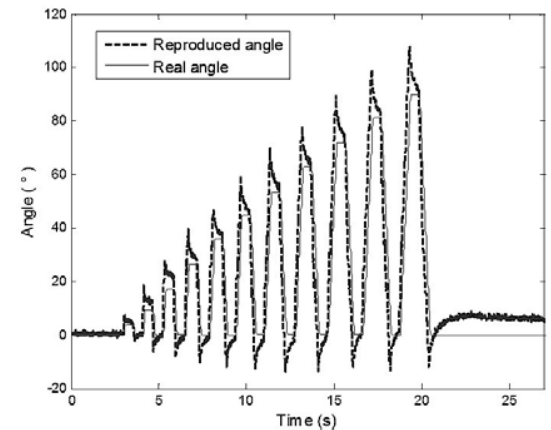

(b)

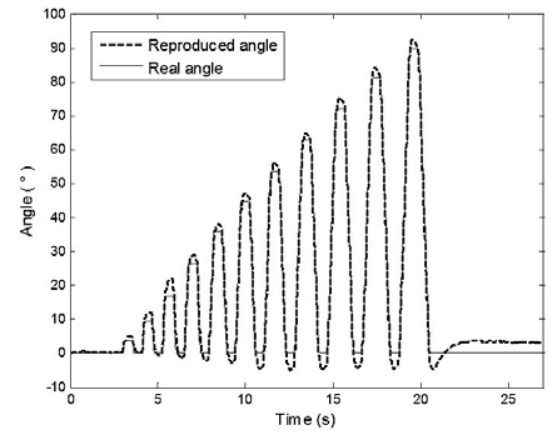

(c)

Fig. 9. Reproduced bending angles from the inverse models of; (a) Model 1, (b) Model 2, and (c) Model 3.

In order to verify the effectiveness of the procedure to estimate the input bending angles using the inverse transfer functions, an arbitrary bending motion was applied to the 
IPMC sensor by rotating the stepper motor shaft by hand. The error values between the real and reproduced bending angles from each inverse transfer function are shown in Table 3. The reproduced bending angle from the inverse transfer function of Model 3 has the smallest error from the real bending angles. Fig. 10 shows the real and reproduced bending angles from the inverse transfer function of Model 3 .

Table 3. Errors from an arbitrary bending motion

\begin{tabular}{llllll}
\hline & Model·1 & Model 2 & Model·3 & Model·4 & Model·5 \\
\hline RMS error & 10.20 & 10.82 & 8.20 & 10.86 & 8.22 \\
\hline
\end{tabular}

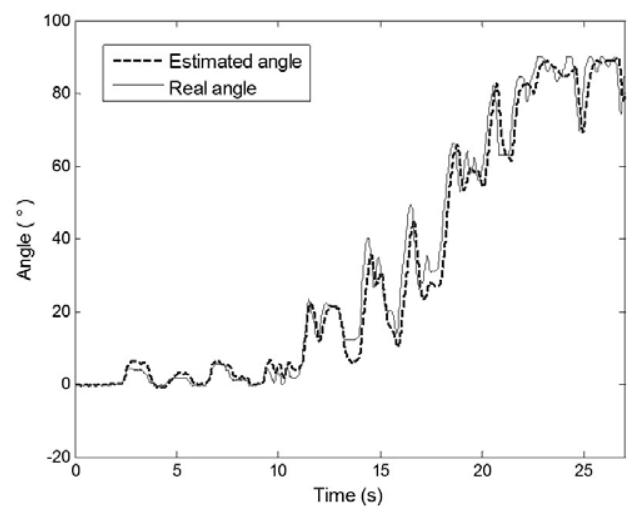

Fig. 10. Reproduced arbitrary bending angle from the inverse model of Model 3.

\section{CONCLUSIONS}

Developing a model tor an IPMC sensor requires a trade-off between simplicity and completeness. If the model is too complex, it is difficult to be implemented to real world applications. One of the advantages of using a circuit model is that the complex composition of a material can be represented as a simple electric circuit in which each component is related to the physical parameter of sensor material.

In the present study, an effective monitoring method of large deflection of an IPMC sensor was investigated using circuit models. Several IPMC circuit models were constructed using simulink in Matlab ${ }^{\circledR}$ and the parameter identification method estimated the parameter values of all models by minimizing the errors between the real and simulated output voltages. The performances of all the models were evaluated by comparing the real with simulated output voltages from the circuit models and the real and reproduced angles from the inverse models. A cascade model connecting unit-circuits in series was introduced. It was found that there were no significant differences in the performances between the unit-circuit models and the corresponding cascade circuit models. All circuit models simulated the output voltages fairly well and the corresponding inverse models also reproduced the input bending angles without significant errors. Nevertheless, the unit-circuit model presented by Kanno et al.[7] showed the best performances among the tested models and has been selected as the most suitable model for simulation of an IPMC sensor.

\section{ACKNOWLEDGMENT}

The authors would like to thank to BME program in SDSM\&T for its Support for this research endeavor.

\section{REFERENCES}

[1] Konyo M, Konishi Y, Tadokoro S, and Kishima T, "Development of velocity sensor using ionic polymermetal composites sensors and actuators," Proc. SPIE, vol. 5385, pp. 307-318, 2004.

[2] Ferrara L, Shahinpoor M, Kim K. J, Schreyer B, Keshavarzi A, Benzel E, and Lantz J, "Use of ionic polymer-metal composites(IPMCs) as a pressure transducer in the human spine," SPIE Conf. Electroactive Polymer Actuators and Devices, vol. 3669, pp. 394-400, 1999.

[3] Biddiss E and Chau T, "Electroactive polymeric sensors in hand prostheses: Bending response of an ionic polymer metal composite," Medical Engineering \& Physics, vol. 28, pp. 568-578, 2006.

[4] Farinholt K and Leo D, "Modeling of electromechanical charge sensing in ionic polymer transducers," Mechanics of Materials, vol. 36, pp. 421-433, 2004.

[5] Chen Z, Tan X, Will A, and Ziel C, “A dynamic model for ionic polymer-metal composite sensors," Smart Materials and Structures, vol. 16, pp. 1477-1488, 2007.

[6] Shahinpoor M and Kim K. J, "The effect of surfaceelectrode resistance on the performance of ionic polymer-metal composite(IPMC) artificial muscles," Smart Materials and Structures, vol. 9, pp. 543-551, 2000.

[7] Kanno R, Tadokoro S, Takamori T, Hattori M, and 
Oguro K, "Linear approximate dynamic model of an ICPF actuator," Proc. IEEE International Conference on Robotics and Automation, vol. 1, pp. 219-225, 1996.

[8] Paquette Jason W, Kim Kwang J, Nam Jae-Do, and Tak Yong S, “An equivalent circuit model for ionic polymer-metal composites and their performance improvement by a clay-based polymer nano-composite technique," Intelligent Material System and Structures, vol. 14, pp. 633-642, 2003.

[9] http://ndeaa.jpl.nasa.gov/nasa-nde/lommas/eap/IPMC PrepProcedure.htm

[10] Bennett M and Leo D, "Ionic liquid as stable solvents for ionic polymer transducers," Sensors and Actuators $A$, vol. 115, pp. 79-90, 2004.

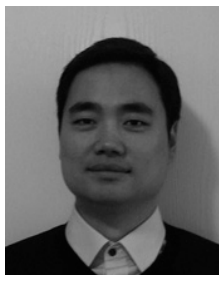

Kiwon Park was born in Busan, Republic of Korea, in 1977. He received the M.S. degree in electrical engineering from the South Dakota School of Mines and Technology, USA, in 2006. He earned PhD degree in biomedical engineering at the same school in 2011. 